\title{
Supplement use during an intergroup clinical trial for breast cancer (S0221)
}

\author{
Gary R. Zirpoli • Patrick M. Brennan • Chi-Chen Hong • Susan E. McCann • \\ Gregory Ciupak - Warren Davis · Joseph M. Unger - G. Thomas Budd • \\ Dawn L. Hershman - Halle C.F. Moore · James Stewart • Claudine Isaacs • \\ Timothy Hobday • Muhammad Salim • Gabriel N. Hortobagyi · Julie R. Gralow • \\ Kathy S. Albain · Christine B. Ambrosone
}

Received: 9 August 2012/Accepted: 22 December 2012/Published online: 10 January 2013

(C) The Author(s) 2013. This article is published with open access at Springerlink.com

\begin{abstract}
The use of supplements during chemotherapy is controversial, partly due to the potential effect of antioxidants on reduced efficacy of chemotherapy-related cytotoxicity. We examined supplement use among breast cancer patients registered to a clinical trial (SWOG 0221) before diagnosis and during treatment. Patients $(n=$ 1,467) completed questionnaires regarding multivitamin and supplement use at trial registration (baseline) to capture use before diagnosis. Of these patients, 1,249 completed a 6-month followup questionnaire to capture use
\end{abstract}

James Stewart-ECOG, Claudine Isaacs-CALGB, Timothy Hobday-NCCTG, Muhammad Salim—CAN (NCIC-CTG)

G. R. Zirpoli · P. M. Brennan - C.-C. Hong ·

S. E. McCann - G. Ciupak · W. Davis · C. B. Ambrosone ( $\square)$ Department of Cancer Prevention and Control, Roswell Park Cancer Institute, Elm \& Carlton Sts, Buffalo, NY 14263, USA e-mail: christine.ambrosone@ roswellpark.org

J. M. Unger

SWOG Statistical Center, Fred Hutchinson Cancer Research

Center, Seattle, WA 98109, USA

G. Thomas Budd · H. C.F.Moore

Department of Solid Tumor Oncology, Cleveland Clinic,

Cleveland, OH 44195, USA

D. L. Hershman

Department of Medicine, Columbia University,

New York, NY 10032, USA

J. Stewart

Department of Medicine, Baystate Medical Center,

Tufts University, Springfield, MA 01199, USA

C. Isaacs

The Fisher Center for Familial Cancer Research, Lombardi

Comprehensive Cancer Center, Washington, DC 20007, USA during treatment. We examined the use of vitamins $\mathrm{C}, \mathrm{D}$, E, B6, B12, folic acid, and calcium at these timepoints, as well as physician recommendations regarding supplement use. The use of vitamins C, E, folic acid, and calcium decreased during treatment, while the use of vitamin B6 increased. Five hundred seventy four patients (51\%) received no physician recommendations regarding supplement use. Among the remaining 49,10\% were advised not to take multivitamins and/or supplements, $7 \%$ were advised to use only multivitamins, and $32 \%$ received recommendations to use multivitamins and/or supplements. Among patients who took vitamin $\mathrm{C}$ before diagnosis, those who were advised not to take supplements were $>5$ times more likely not to use of vitamin $\mathrm{C}$ during treatment

\section{T. Hobday}

Department of Oncology, Mayo Clinic

College of Medicine, Rochester, MN 55905, USA

M. Salim

Allan Blair Cancer Centre, Regina, SK, Canada

G. N. Hortobagyi

Department of Breast Medical Oncology,

The University of Texas MD Anderson Cancer Center,

Houston, TX 77030, USA

J. R. Gralow

Department of Medicine, Seattle Cancer Care Alliance, Fred

Hutchinson Cancer Research Center, Seattle, WA 98109, USA

K. S. Albain

Department of Medicine, Cardinal Bernardin Cancer Center, Loyola University Chicago Stritch School of Medicine, Maywood, IL 60153, USA 
than those not advised to stop use (OR $=5.27,95 \% \mathrm{CI}$ 1.13-24.6). Previous non-users who were advised to take a multivitamin were nearly 5 times more likely to use multivitamins during treatment compared to those who received no recommendation $(\mathrm{OR}=4.66,95 \% \mathrm{CI}$ 2.10-10.3). In this clinical trial for high-risk breast cancer, supplement use generally decreased during treatment. Upon followup from the clinical trial, findings regarding supplement use and survival outcomes will better inform physician recommendations for patients on adjuvant chemotherapy.

Keywords Antioxidants - Dietary supplements . Epidemiology · Breast cancer · Chemotherapy

\section{Introduction}

Dietary supplement use is widespread among adults in the United States [1], and has been increasing over time [2]. Supplement use is especially prevalent among breast cancer patients. In the Women's Healthy Eating and Living study, $58 \%$ of the breast cancer survivors who participated reported use of multivitamins with 46 and $42 \%$ reporting use of vitamins $\mathrm{E}$ and $\mathrm{C}$, respectively $[3,4]$. In the long Island breast cancer study project (LIBCSP), $60 \%$ of patients reported using antioxidant supplements during adjuvant treatment [5]. Breast cancer patients have also been shown to take dietary supplements at dosages well above the recommended daily allowances $[4,5]$.

There is controversy surrounding the use of antioxidant supplements during therapy for cancer, particularly because of their potentially negative effects on treatment efficacy. As previously reviewed [6], a primary mechanism of cytotoxicity for numerous chemotherapy agents, as well as radiation therapy, is through generation of reactive oxygen species (ROS), which damage and kill tumor as well as normal cells. Because antioxidants can reduce ROS and potentially reduce treatment efficacy, it has been recommended that physicians discourage their patients from use of antioxidant supplements during treatment [7]. The American Institute for Cancer Research states that "taking dietary supplements containing levels of nutrients with antioxidant properties much greater than the dietary reference intakes is not recommended during chemotherapy" $[8,9]$, and a 2005 Journal of Clinical Oncology commentary from a large comprehensive cancer center discloses that their patients are discouraged from taking supplements during cancer treatment [10]. However, there are also reports that antioxidants may reduce chemotherapyinduced neurotoxicity and cardiotoxicity [6]. In addition, in the life after cancer epidemiology (LACE) Study, breast cancer patients frequently using vitamin $\mathrm{C}$ and vitamin $\mathrm{E}$ had fewer recurrences and a reduced risk of mortality, but an increased risk of death with frequent carotenoid use [11].

Although supplement use appears to be common among cancer patients, many patients do not discuss supplement use with physicians. Estimates of percentages of patients who report supplement use to their physicians range from as low as $33 \%$ to up to $82 \%$ of patients [12-15]. In addition, studies have indicated that the extent to which health care providers communicate with patients about supplement use is low with lack of confident knowledge on the subject a major contributing factor $[16,17]$.

In this report, we present the characteristics of patients enrolled in an observational study ancillary to a large cooperative group therapeutic trial for women with highrisk breast cancer. With data collected regarding previous habits, use at registration, and again following completion of treatment, this trial provides an ideal basis for examining the use of vitamin supplements before diagnosis and during chemotherapy, and the extent to which discussions with patients' physicians influenced patterns of usage during treatment.

\section{Methods}

\section{Patients}

This study was conducted ancillary to a North American Breast Cancer Group phase III trial (S0221) led by SWOG, formerly Southwest Oncology Group. Eligibility criteria for S0221 included confirmed diagnosis of operable Stage II or III invasive breast cancer with known estrogen or progesterone receptor status, high-risk status based on tumor size or nodal involvement, a history of modified radical mastectomy or local excision of all tumors plus axillary node dissection or sentinel node resection, and no previous history of chemotherapy or radiation. Criteria also included normal blood work, age $18+$, Zubrod performance status of $0-2$, as well as a medical history free of heart failure, angina, HIV, and previous malignancies.

As shown in Fig. 1, patients enrolled in the trial were randomized to four treatment arms. Each arm utilized the same 3 drugs, but followed a different treatment schedule. Patients received either doxorubicin plus cyclophosphamide every 2 weeks with pegfilgrastim support, or weekly doxorubicin plus daily cyclophosphamide with filgrastim support. Patients then received either 12 cycles of weekly paclitaxel or paclitaxel every 2 weeks with pegfilgrastim support for 6 cycles. 
Fig. 1 Design of SWOG 0221, a trial for women with high-risk breast cancer $A$ doxorubicin,

$C$ cyclophosphamide,

$G$ filgrastim, $P E G G$

pegfilgrastim, $T$ paclitaxel

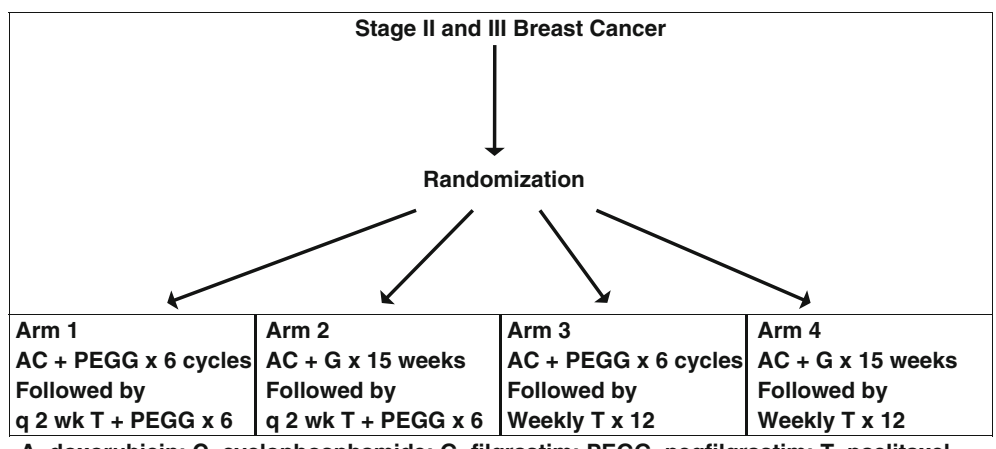

A=doxorubicin; $C=$ =yclophosphamide; $G$ =filgrastim; $P E G G=$ pegfilgrastim; $T=$ paclitaxe
This ancillary study regarding effects of lifestyle factors on treatment outcomes was described in the therapeutic consent for the trial, and informed consent included permission to contact the patient. Upon agreement to participate, patients' contact information was forwarded to research study staff for followup with the potential participants. This study ancillary to S0221 was approved by the Institutional Review Board at Roswell Park Cancer Institute, and at all participating institutions that enrolled patients.

\section{Study design}

The long-term goal of this prospective ancillary study, the diet, exercise, lifestyle, and cancer prognosis (DELCaP) study, is to determine if habits and practices before and during chemotherapy have any impact on treatment outcomes, including treatment-related toxicities as well as recurrence and survival. For this purpose, patients are contacted at registration to the trial, the DELCaP study is described in more detail, and a questionnaire is sent to the participants. This baseline questionnaire gathers data on race and ethnicity, menopausal status, height and weight, histories of smoking, alcohol consumption, and physical activity. A 110-item Food Frequency Questionnaire and detailed data on use of vitamins and supplements are also collected via questions adapted from the VITAL study, which has been extensively validated [18]. Participants are asked to gather all multivitamins and supplements they are taking. For multivitamins, patients may choose from a predefined list of popular brands of multivitamin (Centrum, etc.) or may fill in the specific brand they use, as well as the number of days of use and the number of years taken in the past 10. Patients are then queried for dosages of select multivitamin components. For individual supplement use, frequency of use and daily dose are recorded.

At 6 months after registration to the trial, when chemotherapy should be completed, a second questionnaire is mailed to participants. This questionnaire has the same general format as the first, and updates lifestyle factors and supplement use during treatment. It also includes a section on recommendations received from the patient's physicians, and queries if the use of vitamins and supplements during treatment was discussed and if the participants' doctors made any recommendations. If recommendations were received, patients are further queried regarding suggestions (Fig. 2). Additional questionnaires are sent annually soliciting the same information on lifestyle factors for eventual analysis in relation to recurrence and survival.
Fig. 2 Question at completion of chemotherapy regarding physician recommendations on supplement use during treatment

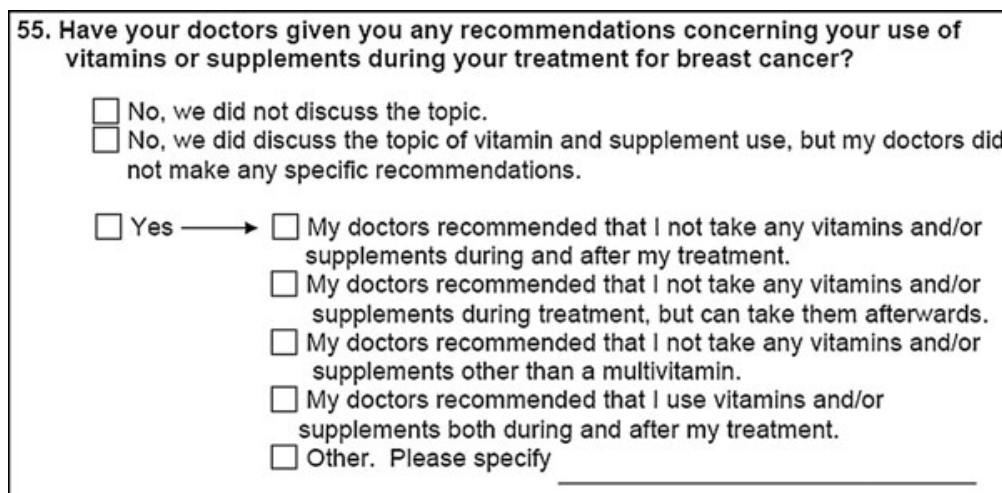


All questionnaires were entered twice by different data entry personnel. This analysis includes 1,467 participants who completed the baseline questionnaire; of these women, we include data from 1,249 patients who also completed the 6-month questionnaire. Two hundred eighteen participants withdrew from the study between the first questionnaire and the second questionnaire. These participants were younger, less likely to be non-Hispanic white, were more likely to have smoked, and had lower education.

\section{Statistical analysis}

The focus of this analysis is on use of vitamin supplements before diagnosis and during treatment for breast cancer, and in relation to physician recommendations. In the first questionnaire, patients were asked if they took supplements before breast cancer diagnosis and/or, if they took supplements after diagnosis (No; Yes, but less than once a week; Yes, at least once a week). In the second questionnaire, participants were asked if they took supplements during treatment. Those who responded that they used supplements at least once per week were classified as "users." "Non-users" were those who did not use at all, or used less than once per week. Because of the low rates of use of other supplements, we present data only on supplements with at least $10 \%$ use at any time point. For all analyses presented, we compared supplement use before diagnosis to supplement use during treatment. Although physician recommendations were not queried until the second questionnaire, it is possible that physician recommendations at diagnosis influenced supplement use during the period between diagnosis and treatment. Thus, we compared use before diagnosis and 6 months following entry into the trial.

Answers regarding physician recommendations were categorized into "No recommendation," "Do not use vitamins and/or supplements during treatment," "Use a multivitamin only," and "Use vitamins and supplements during and after treatment." Participants also had the option to write in their physician's recommendations, instead of choosing from the predefined list. These answers were hand-coded and grouped into one of the first three categories. Of these, written answers from 115 participants could not be classified into any of the categories.

For each supplement, participants were stratified by use before diagnosis into baseline users and non-users. For baseline users, unconditional logistic regression was used to estimate odds ratios (OR) and $95 \%$ confidence intervals (CI) for the association between discontinuing use during treatment and physician recommendations. Analogous analyses for baseline non-users were conducted to model the association between beginning use during treatment and physician recommendations. Self-reported height and usual weight before diagnosis were used to compute body mass index (BMI) in kilograms/meters ${ }^{2}$. ORs and $95 \%$ CIs were calculated and adjusted for age at completion of the first questionnaire, BMI, race/ethnicity, education level, menopausal status, and smoking status (never/former/current). All analyses were conducted by SAS 9.2.

\section{Results}

The baseline characteristics of 1,467 breast cancer patients who completed the first questionnaire are shown in Table 1. The mean age at baseline was 51 years (range: 23-80 years); $80 \%$ of participants identified themselves as non-Hispanic white and the majority of patients (71\%) were overweight or obese. Slightly more than half were postmenopausal. More than half $(56 \%)$ were never smokers, $72 \%$ had at least some college, and the majority were married or living as married $(73 \%)$.

Supplements with usage of at least $10 \%$ of the study population either before diagnosis, or during treatment for breast cancer, are displayed in Table 2. Multivitamins were the supplements used most frequently with $48 \%$ use before diagnosis and $43 \%$ use during treatment. Other supplements that showed decreases in use during treatment included vitamin $\mathrm{C}(20 \%$ before diagnosis vs. $12 \%$ during treatment), vitamin E (15 vs. $6 \%$ ), folic acid (10 vs. $8 \%$ ), calcium (34 vs. $28 \%$ ), fish oil, EPA, omega-3, flaxseed or cod liver oil (22 vs. $12 \%$ ), and glucosamine (13 vs. $7 \%$ ). Compared to before diagnosis, vitamin B6 use increased during treatment (10 vs. $19 \%$ ).

Average supplement doses per day before diagnosis and during breast cancer treatment are presented in Table 3 . After diagnosis and during treatment, vitamin $\mathrm{D}$ use increased overall among those women in the middle and highest categories of use with a concomitant decrease in the lowest category of use. As expected, use of higher doses of vitamin B6 (13+ mg/day) increased markedly during treatment, from $50 \%$ before diagnosis to $87 \%$ during treatment. While overall use of B12 decreased slightly between during breast cancer treatment, the percentage of high average daily dosage $(24+\mu \mathrm{g} /$ day $)$ increased from $67 \%$ before diagnosis to $84 \%$ during treatment. Calcium use was largely unchanged from before diagnosis to during treatment. Iron intake increased during treatment in the lowest and highest categories, but decreased for the middle category. There were no clear trends for vitamins $\mathrm{C}$ and $\mathrm{E}$.

Table 4 shows data collected from the 2nd questionnaire administered at 6 months related to physician recommendations regarding supplement use. Five hundred seventy four patients $(51 \%)$ received no recommendation (either due to no discussion of supplement use with their doctors, or did discuss, but received no specific recommendations). Among the remaining 560 participants who did receive recommendations, 
Table 1 Characteristics of 1,467 DELCaP participants

\begin{tabular}{|c|c|}
\hline Characteristic & $N(\%)$ \\
\hline \multicolumn{2}{|l|}{ Age } \\
\hline$<35$ & $63(4.3)$ \\
\hline $35-39$ & $109(7.4)$ \\
\hline $40-44$ & $195(13.3)$ \\
\hline $45-49$ & $276(18.8)$ \\
\hline $50-54$ & $277(18.9)$ \\
\hline $55-59$ & $225(15.3)$ \\
\hline $60-64$ & $174(11.9)$ \\
\hline $65-69$ & $100(6.8)$ \\
\hline$\geq 70$ & $48(3.3)$ \\
\hline \multicolumn{2}{|l|}{ Race } \\
\hline Non-hispanic white & $1,176(80.2)$ \\
\hline Hispanic & $65(4.4)$ \\
\hline Black/African American & $104(7.1)$ \\
\hline Other & $122(8.3)$ \\
\hline \multicolumn{2}{|l|}{ BMI } \\
\hline Underweight $(\leq 18.5)$ & $12(0.8)$ \\
\hline Normal (18.5-24.9) & $412(28.4)$ \\
\hline Overweight (25-29.9) & $469(32.4)$ \\
\hline Obese $(\geq 30)$ & $556(38.4)$ \\
\hline \multicolumn{2}{|l|}{ Menopausal status } \\
\hline Premenopausal & $686(46.8)$ \\
\hline Postmenopausal & $781(53.2)$ \\
\hline \multicolumn{2}{|l|}{ Smoking history } \\
\hline Never smokers & $806(55.5)$ \\
\hline Former smokers & $461(31.7)$ \\
\hline Current smokers & $186(12.8)$ \\
\hline \multicolumn{2}{|l|}{ Education } \\
\hline Grade school or some high school & $97(6.6)$ \\
\hline High school graduate or GED & $311(21.3)$ \\
\hline Some college or technical school & $522(35.8)$ \\
\hline College graduate & $325(22.3)$ \\
\hline Advanced degree & $205(14.0)$ \\
\hline \multicolumn{2}{|l|}{ Marital status } \\
\hline Married or living as married & $1,067(73.1)$ \\
\hline Widowed & $68(4.6)$ \\
\hline Separated or Divorced & $236(16.2)$ \\
\hline Single & $89(6.1)$ \\
\hline
\end{tabular}

112 received recommendations not to take multivitamins and/ or supplements $(10 \%), 80$ received recommendations to use only multivitamins ( $7 \%$ ), and 368 received recommendations to use multivitamins and/or supplements (32\%).

Multivitamin and supplement use during treatment among baseline users

Multivitamin and supplement use during treatment was compared to use before diagnosis in relation to physician recommendations with the reference group comprised of those receiving no recommendations. Models for baseline multivitamin and supplement users adjusted for age at baseline alone and additionally adjusted for BMI at baseline, race/ethnicity, education level, menopausal status, and smoking status are presented in Table 5. Among those using multivitamins at least once per week at baseline, patients receiving recommendations not to take multivitamins and/or supplements were 45 times more likely to reduce multivitamin use to less than once per week during treatment, compared to those receiving no recommendations (adjusted odds ratio $(\mathrm{aOR})=45.0,95 \% \quad \mathrm{CI}$ 10.6-191). Patients who were advised to use multivitamins only were less likely to decrease use than those receiving no recommendations $(\mathrm{aOR}=0.21,95 \% \mathrm{CI} 0.08-0.6)$, as were patients receiving recommendations to use multivitamins and/or supplements $(\mathrm{aOR}=0.46,95 \%$ CI 0.29 $0.72)$.

Among those using vitamin $\mathrm{C}$ supplements at least once per week at baseline, all patients receiving recommendations not to take multivitamins and/or supplements decreased use of vitamin $\mathrm{C}$ to less than once per week. Patients who were told to use multivitamins only were over 5 times more likely to decrease use of vitamin C, compared to those receiving no recommendations $(\mathrm{aOR}=5.27,95 \% \mathrm{CI}=1.13-24.6)$. Compared to those receiving no advice, participants receiving recommendations to use multivitamins and/or supplements during treatment were less likely to decrease vitamin $\mathrm{C}$ use to less than once per week although this difference was not statistically significant $(\mathrm{aOR}=0.72,95 \% \mathrm{CI}=0.37-$ 1.39).

Among those using vitamin E supplements at least once per week at baseline, all patients receiving recommendations not to take multivitamins and/or supplements decreased use of vitamin $\mathrm{E}$ to less than once per week. Those receiving recommendations to use multivitamins only were 3.5 times as likely to decrease vitamin $\mathrm{E}$ use to less than once per week compared to those receiving no recommendations $\quad(\mathrm{aOR}=3.52,95 \%$ CI $0.40-31.3)$. Patients receiving recommendations to use multivitamins and/or supplements during treatment were less likely to decrease use of vitamin $\mathrm{E}$ to less than once per week than those receiving no recommendations $(\mathrm{aOR}=0.48,95 \%$ CI 0.20-1.17).

Use of the other supplements assessed showed similar trends in patterns of use although there were small numbers for some subgroups. Fish oil and glucosamine users at baseline, in particular, who received recommendations to not use vitamins or supplements were much more likely to stop usage, compared to those receiving no recommendation $(\mathrm{aOR}=25.3,95 \%$ CI 3.30-195; aOR $=12.3,95 \%$ CI 1.48-102, respectively). 
Table 2 Supplement use before and during breast cancer treatment

\begin{tabular}{|c|c|c|c|c|c|}
\hline \multirow{2}{*}{$\begin{array}{l}\text { Table } 2 \text { Supplement use } \\
\text { before and during breast cancer } \\
\text { treatment }\end{array}$} & \multirow[t]{2}{*}{ Supplement use before diagnosis } & \multicolumn{2}{|c|}{ Supplement use during treatment } & \multirow{2}{*}{$\begin{array}{l}\text { Total } \\
N(\%)\end{array}$} & \multirow[t]{2}{*}{$P^{\mathrm{a}}$} \\
\hline & & $\begin{array}{l}\text { No } \\
N(\%)\end{array}$ & $\begin{array}{l}\text { Yes } \\
N(\%)\end{array}$ & & \\
\hline & \multicolumn{5}{|l|}{ Multivitamin } \\
\hline & No & $510(41.2)$ & $133(10.7)$ & $643(51.9)$ & 0.0009 \\
\hline & Yes & $193(15.6)$ & $402(32.5)$ & $595(48.1)$ & \\
\hline & Total & $703(56.8)$ & $535(43.2)$ & $1,238(100)$ & \\
\hline & \multicolumn{5}{|l|}{ Vitamin $\mathrm{C}$} \\
\hline & No & $931(75.2)$ & $62(5.0)$ & $993(80.2)$ & $<.0001$ \\
\hline & Yes & $160(12.9)$ & $85(6.9)$ & $245(19.8)$ & \\
\hline & Total & $1,091(88.1)$ & $147(11.9)$ & $1,238(100)$ & \\
\hline & \multicolumn{5}{|l|}{ Vitamin D } \\
\hline & No & $795(64.2)$ & $155(12.5)$ & $950(76.7)$ & 0.1229 \\
\hline & Yes & $129(10.4)$ & $160(12.9)$ & $289(23.3)$ & \\
\hline & Total & 924 (74.6) & $315(25.4)$ & $1,239(100)$ & \\
\hline & \multicolumn{5}{|l|}{ Vitamin E } \\
\hline & No & $1,005(81.2)$ & $42(3.4)$ & $1,047(84.6)$ & $<.0001$ \\
\hline & Yes & $154(12.4)$ & $37(3.0)$ & $191(15.4)$ & \\
\hline & Total & 1,159 (93.6) & $79(6.4)$ & $1,238(100)$ & \\
\hline & \multicolumn{5}{|l|}{ Vitamin B6 } \\
\hline & No & $933(75.4)$ & $186(15.0)$ & $1,119(90.4)$ & $<.0001$ \\
\hline & Yes & $69(5.6)$ & $50(4.0)$ & $119(9.6)$ & \\
\hline & Total & $1,002(80.9)$ & $236(19.1)$ & $1238(100)$ & \\
\hline & \multicolumn{5}{|l|}{ Folic Acid } \\
\hline & No & $1,051(84.8)$ & $62(5.0)$ & $1,113(89.8)$ & 0.0231 \\
\hline & Yes & $90(7.3)$ & $37(3.0)$ & $127(10.2)$ & \\
\hline & Total & $1,141(92)$ & $99(8)$ & $1,240(100)$ & \\
\hline & \multicolumn{5}{|l|}{ Vitamin B12 } \\
\hline & No & $999(80.5)$ & $82(6.6)$ & $1,081(87.1)$ & 0.2622 \\
\hline & Yes & $97(7.8)$ & $63(5.1)$ & $160(12.9)$ & \\
\hline & Total & $1,096(88.3)$ & $145(11.7)$ & $1,241(100)$ & \\
\hline & \multicolumn{5}{|l|}{ Calcium } \\
\hline & No & $695(56.0)$ & $119(9.6)$ & 814 (65.6) & $<.0001$ \\
\hline & Yes & $198(16.0)$ & $229(18.4)$ & $427(34.4)$ & \\
\hline & Total & $893(72)$ & $348(28)$ & $1,241(100)$ & \\
\hline & \multicolumn{5}{|c|}{ Fish oil, EPA, omega-3, flaxseed or cod liver oil } \\
\hline & No & $907(73.5)$ & $47(3.8)$ & $954(77.3)$ & $<.0001$ \\
\hline & Yes & $172(13.9)$ & $108(8.8)$ & $280(22.7)$ & \\
\hline & Total & $1,079(87.4)$ & 155 (12.6) & $1,234(100)$ & \\
\hline & \multicolumn{5}{|l|}{ Glucosamine } \\
\hline & No & $1,059(85.7)$ & $18(1.5)$ & $1,077(87.1)$ & $<.0001$ \\
\hline${ }^{a}$ McNemar's test comparing the & Yes & $97(7.8)$ & $62(5.0)$ & $159(12.9)$ & \\
\hline ices in use before and during & Total & $1,156(93.5)$ & $80(6.5)$ & $1,236(100)$ & \\
\hline
\end{tabular}

${ }^{\text {a }}$ McNemar's test comparing the differences in use before and during treatment
Multivitamin and/or supplement use during treatment among non-users

Analogous models for baseline non-users of multivitamins and/or supplements are presented in Table 6. Multivitamin use during treatment among previous non-users increased among those whose physicians recommended multivitamin use only during treatment $(\mathrm{aOR}=4.66,95 \%$ CI 2.10-10.3). Few participants who were non-users of vitamin $\mathrm{C}$ or vitamin $\mathrm{E}$ at baseline began using these vitamins 
Table 3 Average supplement dose per day before and during breast cancer treatment

\begin{tabular}{|c|c|c|}
\hline \multirow[t]{2}{*}{ Supplement } & \multicolumn{2}{|l|}{ Time period } \\
\hline & $\begin{array}{l}\text { Before diagnosis } \\
N(\%)\end{array}$ & $\begin{array}{l}\text { During treatment } \\
N(\%)\end{array}$ \\
\hline \multicolumn{3}{|c|}{ Vitamin C (mg/day) } \\
\hline$<75$ & $19(8)$ & $15(10.3)$ \\
\hline $75-750$ & $142(59.9)$ & $81(55.9)$ \\
\hline $750+$ & $76(32.1)$ & $49(33.8)$ \\
\hline \multicolumn{3}{|c|}{ Vitamin D ( $\mu \mathrm{g} /$ day $)$} \\
\hline$<15$ & $147(52.3)$ & $137(44.9)$ \\
\hline $15-150$ & $129(45.9)$ & $158(51.8)$ \\
\hline $150+$ & $5(1.8)$ & $11(3.6)$ \\
\hline \multicolumn{3}{|c|}{ Vitamin E (mg/day) } \\
\hline$<15$ & $1(0.6)$ & $4(5.1)$ \\
\hline $15-150$ & $64(36.8)$ & $29(37.2)$ \\
\hline $150+$ & $109(62.6)$ & $45(57.7)$ \\
\hline \multicolumn{3}{|c|}{ Vitamin B6 (mg/day) } \\
\hline$<1.3$ & $3(2.8)$ & $1(0.5)$ \\
\hline $1.3-13$ & $51(47.7)$ & $27(12.3)$ \\
\hline $13+$ & $53(49.5)$ & $191(87.2)$ \\
\hline \multicolumn{3}{|c|}{ Folic acid ( $\mu \mathrm{g} /$ day) } \\
\hline$<400$ & $38(34.2)$ & $28(30.1)$ \\
\hline $400+$ & $73(65.8)$ & $65(69.9)$ \\
\hline \multicolumn{3}{|c|}{ Vitamin B12 ( $\mu \mathrm{g} /$ day) } \\
\hline$<2.4$ & 0 & 0 \\
\hline $2.4-24$ & $47(33.3)$ & $22(15.9)$ \\
\hline $24+$ & $94(66.7)$ & $116(84.1)$ \\
\hline \multicolumn{3}{|c|}{ Calcium (mg/day) } \\
\hline$<1000$ & $265(65.4)$ & $232(66.1)$ \\
\hline $1000+$ & $140(34.6)$ & $119(33.9)$ \\
\hline
\end{tabular}

Table 4 Patient-reported physician recommendations on vitamin and supplement use during treatment

\begin{tabular}{lc}
\hline Physician recommendation & $N(\%)$ \\
\hline No recommendation & $574(50.6)$ \\
Do not use vitamins or supplements during treatment & $112(9.9)$ \\
Use a multivitamin only & $80(7.1)$ \\
Use vitamins and supplements during and after treatment & $368(32.4)$ \\
Unable to be classified & 115 \\
\hline
\end{tabular}

during treatment (6 and $5 \%$, respectively), regardless of recommendations received. For non-users of vitamin $\mathrm{C}$ at baseline, participants receiving recommendations to use vitamins and supplements during treatment were twice as likely to use vitamin $\mathrm{C}$ during treatment $(\mathrm{aOR}=1.82$, $95 \%$ CI 0.99-3.33); similar associations were observed for vitamin $\mathrm{E}(\mathrm{aOR}=4.59,95 \%$ CI 2.10-10.0). Baseline non-users of vitamin $\mathrm{D}$ receiving recommendations to use vitamins and supplements were nearly four times more likely to start vitamin $\mathrm{D}$ use than those receiving no recommendation $(\mathrm{aOR}=3.86,95 \%$ CI 2.55-5.86). Similar associations were observed in vitamins B6 and B12 $(\mathrm{aOR}=6.39,95 \% \mathrm{CI} 4.24-9.62 ; \mathrm{aOR}=5.10,95 \% \mathrm{CI}$ 2.78-9.37, respectively).

\section{Discussion}

Our analyses of multivitamin and supplement use during chemotherapy by women enrolled in this clinical trial for high-risk breast cancer indicate that, in general, supplement use decreased during chemotherapy. However, use of vitamin B6 increased, and while the percentage of vitamin B12 users decreased slightly, more users reported vitamin B12 use at a high dosage during treatment than before diagnosis, perhaps to alleviate side effects of chemotherapy including neuropathy and anemia. Use of vitamin D also increased during treatment.

Supplement use in our study differed from previously published results. It has been reported that approximately $45-80 \%$ of breast cancer patients in the United States use antioxidant supplements, including during treatment [19]. In the LIBCSP, $60.5 \%$ of breast cancer patients reported using supplements during treatment, and $38 \%$ reported using antioxidants during chemotherapy [5]. Of antioxidant users, $69 \%$ used doses higher than those contained in a multivitamin. In our study of participants in a Cooperative Group clinical trial, the use of supplements was much lower at baseline $(20 \%$ used vitamin $\mathrm{C}$ at least once per week, $15 \%$ used vitamin $\mathrm{E}$ at least once per week) and particularly lower during the course of chemotherapy (12\% used vitamin $\mathrm{C}$ at least once per week, $8 \%$ used vitamin $\mathrm{E}$ at least once per week). This unexpected finding of low use at baseline could be due to temporal changes in awareness with growing publicity and recommendations that supplements may reduce treatment efficacy. The source populations may differ as well. Participants in both the LIBCSP and LACE studies were older on average than participants in our study (58.3 and 56.3 years versus 51.3 , respectively) $[5,11]$. Women were enrolled onto S0221 at sites throughout the country, from large metropolitan cancer centers to community oncology group programs in more rural areas, which may suggest that our sample is more representative of the general population in the U.S. Supplement use may be lower because the patients in this study had high-risk breast cancer, and may be less willing to use something with unknown effects on cancer therapy.

Despite the body of data from laboratory and preclinical studies demonstrating that supplements may interfere with chemotherapeutic efficacy, it is still unclear if use of supplements during treatment has any effect on therapeutic 
Table 5 Odds of discontinuing supplement use during treatment among baseline multivitamin and antioxidant supplement users, according to physician recommendations

\begin{tabular}{|c|c|c|c|}
\hline \multirow[t]{2}{*}{ Physician advice } & \multicolumn{3}{|c|}{ Usage during treatment ${ }^{\mathrm{a}}$} \\
\hline & $\%$ Who stop use & $\mathrm{OR}^{\mathrm{b}}(95 \% \mathrm{CI})$ & $\mathrm{OR}^{\mathrm{c}}(95 \% \mathrm{CI})$ \\
\hline \multicolumn{4}{|c|}{ Multivitamin supplement use $>1$ /week at baseline $(N=542)$} \\
\hline No recommendation & $33 \%(81 / 243)$ & 1.0 (ref) & 1.0 (ref) \\
\hline Do not use vitamins or supplements & $96 \%(47 / 49)$ & $45.0(10.6-191)$ & $44.7(10.5-190)$ \\
\hline Use a multivitamin only & $10 \%(5 / 49)$ & $0.21(0.08-0.56)$ & $0.22(0.08-0.60)$ \\
\hline Use vitamins and supplements & $18 \%(37 / 201)$ & $0.44(0.28-0.69)$ & $0.46(0.29-0.72)$ \\
\hline \multicolumn{4}{|c|}{ Vitamin C supplement use $>1$ /week at baseline $(N=214)$} \\
\hline No recommendation & $60 \%(55 / 91)$ & 1.0 (ref) & 1.0 (ref) \\
\hline Do not use vitamins or supplements & $100 \%(28 / 28)$ & NA & NA \\
\hline Use a multivitamin only & $91 \%(20 / 22)$ & $5.99(1.31-27.5)$ & $5.27(1.13-24.6)$ \\
\hline Use vitamins and supplements & $51 \%(37 / 73)$ & $0.69(0.36-1.29)$ & $0.72(0.37-1.39)$ \\
\hline \multicolumn{4}{|c|}{ Vitamin D supplement use $>1$ week at baseline $(N=259)$} \\
\hline No recommendation & $47 \%(53 / 112)$ & 1.0 (ref) & 1.0 (ref) \\
\hline Do not use vitamins or supplements & $72 \%(18 / 25)$ & $2.76(1.06-7.24)$ & $2.61(0.97-6.99)$ \\
\hline Use a multivitamin only & $58 \%(11 / 19)$ & $1.48(0.54-4.08)$ & $1.39(0.48-3.99)$ \\
\hline Use vitamins and supplements & $36 \%(37 / 103)$ & $0.66(0.38-1.16)$ & $0.65(0.37-1.16)$ \\
\hline \multicolumn{4}{|c|}{ Vitamin E supplement use $>1$ week at baseline $(N=169)$} \\
\hline No recommendation & $79 \%(59 / 75)$ & 1.0 (ref) & 1.0 (ref) \\
\hline Do not use vitamins or supplements & $100 \%(21 / 21)$ & NA & NA \\
\hline Use a multivitamin only & $94 \%(16 / 17)$ & $3.74(0.45-30.8)$ & $3.52(0.40-31.3)$ \\
\hline Use vitamins and supplements & $70 \%(39 / 56)$ & $0.58(0.26-1.30)$ & $0.48(0.20-1.17)$ \\
\hline \multicolumn{4}{|c|}{ Vitamin B6 supplement use $>1$ /week at baseline $(N=102)$} \\
\hline No recommendation & $62 \%(26 / 42)$ & 1.0 (ref) & 1.0 (ref) \\
\hline Do not use vitamins or supplements & $90 \%(9 / 10)$ & $5.50(0.62-49.1)$ & $11.0(0.95-126)$ \\
\hline Use a multivitamin only & $75 \%(9 / 12)$ & $1.06(0.22-5.00)$ & $1.86(0.25-14.0)$ \\
\hline Use vitamins and supplements & $42 \%(16 / 38)$ & $0.36(0.14-0.93)$ & $0.30(0.09-0.98)$ \\
\hline \multicolumn{4}{|c|}{ Folic acid supplement use $>1 /$ week at baseline $(N=111)$} \\
\hline No recommendation & $72 \%(31 / 43)$ & 1.0 (ref) & 1.0 (ref) \\
\hline Do not use vitamins or supplements & $100 \%(13 / 13)$ & NA & NA \\
\hline Use a multivitamin only & $89 \%(8 / 9)$ & $3.01(0.33-27.1)$ & $3.04(0.30-31.2)$ \\
\hline Use vitamins and supplements & $61 \%(28 / 46)$ & $0.65(0.26-1.60)$ & $0.66(0.24-1.80)$ \\
\hline \multicolumn{4}{|c|}{ Vitamin B12 supplement use $>1$ /week at baseline $(N=143)$} \\
\hline No recommendation & $58 \%(36 / 62)$ & 1.0 (ref) & 1.0 (ref) \\
\hline Do not use vitamins or supplements & $91 \%(10 / 11)$ & $7.48(0.88-63.2)$ & $7.83(0.91-67.1)$ \\
\hline Use a multivitamin only & $64 \%(7 / 11)$ & $1.01(0.25-4.03)$ & $1.17(0.27-5.11)$ \\
\hline Use vitamins and supplements & $56 \%(33 / 59)$ & $0.89(0.43-1.87)$ & $0.87(0.40-1.91)$ \\
\hline \multicolumn{4}{|c|}{ Calcium supplement use $>1$ /week at baseline $(N=388)$} \\
\hline No recommendation & $53 \%(87 / 165)$ & 1.0 (ref) & 1.0 (ref) \\
\hline Do not use vitamins or supplements & $83 \%(34 / 41)$ & $4.22(1.77-10.1)$ & $4.15(1.70-10.1)$ \\
\hline Use a multivitamin only & $47 \%(16 / 34)$ & $0.78(0.37-1.64)$ & $0.93(0.43-2.00)$ \\
\hline Use vitamins and supplements & $33 \%(49 / 148)$ & $0.44(0.28-0.70)$ & $0.48(0.30-0.77)$ \\
\hline \multicolumn{4}{|c|}{ Fish oil, EPA, omega-3, flaxseed, or cod liver oil use $>1 /$ week at baseline $(N=242)$} \\
\hline No recommendation & $57 \%(64 / 113)$ & 1.0 (ref) & 1.0 (ref) \\
\hline Do not use vitamins or supplements & $97 \%(31 / 32)$ & $24.5(3.22-187)$ & $25.3(3.30-195)$ \\
\hline Use a multivitamin only & $67 \%(12 / 18)$ & $1.66(0.57-4.81)$ & $1.47(0.49-4.37)$ \\
\hline Use vitamins and supplements & $47 \%(37 / 79)$ & $0.72(0.40-1.29)$ & $0.72(0.39-1.33)$ \\
\hline \multicolumn{4}{|c|}{ Glucosamine supplement use $>1$ /week at baseline $(N=142)$} \\
\hline No recommendation & $58 \%(35 / 60)$ & 1.0 (ref) & 1.0 (ref) \\
\hline Do not use vitamins or supplements & $95 \%(19 / 20)$ & $11.8(1.47-95.1)$ & $12.3(1.48-102)$ \\
\hline Use a multivitamin only & $73 \%(8 / 11)$ & $1.77(0.42-7.50)$ & $1.64(0.37-7.22)$ \\
\hline Use vitamins and supplements & $47 \%(24 / 51)$ & $0.60(0.28-1.30)$ & $0.61(0.27-1.36)$ \\
\hline
\end{tabular}

${ }^{a}$ Continue use defined as those consuming the supplement $\geq$ once per week; Stop use are those consuming the supplement $<$ once per week

${ }^{\mathrm{b}}$ Adjusted for age at baseline

${ }^{\mathrm{c}}$ Adjusted for age at baseline, body mass index at baseline, race/ethnicity, education, menopausal status, and smoking status 
Table 6 Odds of supplement usage during treatment among non-users of multivitamin and antioxidant supplements at baseline, according to physician recommendations

\begin{tabular}{|c|c|c|c|}
\hline Physician advice & $\begin{array}{l}\text { Usage during treatment }{ }^{\mathrm{a}} \\
\% \text { Who start use }\end{array}$ & $\mathrm{OR}^{\mathrm{b}}(95 \% \mathrm{CI})$ & $\mathrm{OR}^{\mathrm{c}}(95 \% \mathrm{CI})$ \\
\hline \multicolumn{4}{|c|}{ Multivitamin supplement non-users $(N=583)$} \\
\hline No recommendation & $14 \%(45 / 327)$ & 1.0 (ref) & 1.0 (ref) \\
\hline Do not use vitamins or supplements & $8 \%(5 / 62)$ & $0.55(0.21-1.43)$ & $0.54(0.21-1.44)$ \\
\hline Use a multivitamin only & $43 \%(13 / 30)$ & $4.74(2.15-10.4)$ & $4.66(2.10-10.3)$ \\
\hline Use vitamins and supplements & $37 \%(61 / 164)$ & $3.70(2.37-5.79)$ & $3.59(2.28-5.66)$ \\
\hline \multicolumn{4}{|l|}{ Vitamin C supplement non-users $(N=910)$} \\
\hline No recommendation & $5 \%(23 / 475)$ & 1.0 (ref) & 1.0 (ref) \\
\hline Do not use vitamins or supplements & $4 \%(3 / 84)$ & $0.74(0.22-2.54)$ & $0.87(0.25-3.03)$ \\
\hline Use a multivitamin only & $3 \%(2 / 58)$ & $0.70(0.16-3.06)$ & $0.69(0.16-3.05)$ \\
\hline Use vitamins and supplements & $9 \%(26 / 293)$ & $1.92(1.07-3.43)$ & $1.82(0.99-3.33)$ \\
\hline \multicolumn{4}{|c|}{ Vitamin D supplement non-users $(N=866)$} \\
\hline No recommendation & $10 \%(44 / 454)$ & 1.0 (ref) & 1.0 (ref) \\
\hline Do not use vitamins or supplements & $5 \%(4 / 87)$ & $0.46(0.16-1.33)$ & $0.47(0.16-1.34)$ \\
\hline Use a multivitamin only & $10 \%(6 / 61)$ & $1.03(0.42-2.53)$ & $0.93(0.38-2.31)$ \\
\hline Use vitamins and supplements & $30 \%(79 / 264)$ & $4.08(2.71-6.15)$ & $3.86(2.55-5.86)$ \\
\hline \multicolumn{4}{|l|}{ Vitamin E supplement non-users $(N=956)$} \\
\hline No recommendation & $2 \%(9 / 493)$ & 1.0 (ref) & 1.0 (ref) \\
\hline Do not use vitamins or supplements & $3 \%(3 / 91)$ & $1.75(0.46-6.61)$ & $1.73(0.45-6.64)$ \\
\hline Use a multivitamin only & $0 \%(0 / 63)$ & NA & NA \\
\hline Use vitamins and supplements & $9 \%(27 / 309)$ & $5.18(2.4-11.2)$ & $4.59(2.10-10.0)$ \\
\hline \multicolumn{4}{|c|}{ Vitamin B6 supplement non-users $(N=1023)$} \\
\hline No recommendation & $7 \%(38 / 526)$ & 1.0 (ref) & 1.0 (ref) \\
\hline Do not use vitamins or supplements & $15 \%(15 / 102)$ & $2.26(1.19-4.29)$ & $2.40(1.25-4.59)$ \\
\hline Use a multivitamin only & $7 \%(5 / 67)$ & $1.04(0.39-2.73)$ & $0.98(0.37-2.60)$ \\
\hline Use vitamins and supplements & $33 \%(109 / 328)$ & $6.41(4.29-9.59)$ & $6.39(4.24-9.62)$ \\
\hline \multicolumn{4}{|c|}{ Folic acid supplement non-users $(N=1016)$} \\
\hline No recommendation & $3 \%(18 / 525)$ & 1.0 (ref) & 1.0 (ref) \\
\hline Do not use vitamins or supplements & $2 \%(2 / 99)$ & $0.60(0.14-2.62)$ & $0.59(0.13-2.61)$ \\
\hline Use a multivitamin only & $3 \%(2 / 71)$ & $0.83(0.19-3.64)$ & $0.85(0.19-3.75)$ \\
\hline Use vitamins and supplements & $9 \%(30 / 321)$ & $2.94(1.61-5.37)$ & $2.88(1.56-5.33)$ \\
\hline \multicolumn{4}{|c|}{ Vitamin B12 supplement non-users $(N=984)$} \\
\hline No recommendation & $3 \%(16 / 507)$ & 1.0 (ref) & 1.0 (ref) \\
\hline Do not use vitamins or supplements & $4 \%(4 / 101)$ & $1.30(0.42-3.98)$ & $1.31(0.42-4.07)$ \\
\hline Use a multivitamin only & $4 \%(3 / 69)$ & $1.40(0.40-4.93)$ & $1.39(0.39-4.95)$ \\
\hline Use vitamins and supplements & $14 \%(43 / 307)$ & $5.02(2.77-9.10)$ & $5.10(2.78-9.37)$ \\
\hline \multicolumn{4}{|l|}{ Calcium supplement non-users $(N=739)$} \\
\hline No recommendation & $9 \%(38 / 403)$ & 1.0 (ref) & 1.0 (ref) \\
\hline Do not use vitamins or supplements & $7 \%(5 / 71)$ & $0.77(0.29-2.03)$ & $0.80(0.30-2.13)$ \\
\hline Use a multivitamin only & $7 \%(3 / 46)$ & $0.70(0.21-2.36)$ & $0.65(0.19-2.23)$ \\
\hline Use vitamins and supplements & $27 \%(60 / 219)$ & $3.80(2.42-5.98)$ & $3.56(2.24-5.66)$ \\
\hline \multicolumn{4}{|c|}{ Fish oil, EPA, omega-3, flaxseed or cod liver oil non-users $(N=880)$} \\
\hline No recommendation & $4 \%(18 / 452)$ & 1.0 (ref) & 1.0 (ref) \\
\hline Do not use vitamins or supplements & $3 \%(2 / 79)$ & $0.61(0.14-2.67)$ & $0.72(0.16-3.23)$ \\
\hline Use a multivitamin only & $3 \%(2 / 62)$ & $0.79(0.18-3.48)$ & $0.74(0.17-3.31)$ \\
\hline Use vitamins and supplements & $7 \%(21 / 287)$ & $1.89(0.99-3.61)$ & $1.91(0.99-3.69)$ \\
\hline \multicolumn{4}{|c|}{ Glucosamine supplement non-users $(N=982)$} \\
\hline No recommendation & $1 \%(6 / 508)$ & 1.0 (ref) & 1.0 (ref) \\
\hline Do not use vitamins or supplements & $1 \%(1 / 91)$ & $0.89(0.11-7.47)$ & $0.78(0.09-6.71)$ \\
\hline Use a multivitamin only & $1 \%(1 / 69)$ & $1.20(0.14-10.1)$ & $1.21(0.14-10.4)$ \\
\hline Use vitamins and supplements & $3 \%(9 / 314)$ & $2.46(0.87-6.98)$ & $2.36(0.82-6.76)$ \\
\hline
\end{tabular}

${ }^{\text {a }}$ Started use defined as those consuming the supplement $\geq$ once per week; Continued non-use are those consuming the supplement $<$ once per week

b Adjusted for age at baseline

c Adjusted for age at baseline, body mass index at baseline, race/ethnicity, education, menopausal status, and smoking status 
outcomes, as few data are available. In a prospective study of 1,038 patients with stage III colon cancer enrolled in the NCIsponsored Cancer and Leukemia Group B, Ng and colleagues found no significant survival benefit to multivitamin use during treatment [20]. In a review of 22 peer-reviewed studies, Greenlee et al. [19] concluded that "findings did not support any conclusions regarding the effects of individual antioxidant supplements during conventional breast cancer treatment on toxicities, tumor response, recurrence, or survival." Data from the Shanghai Breast Cancer Survival Study, a large cohort of breast cancer patients with 532 recurrences and 444 deaths, showed that use of vitamin E, vitamin C and multivitamins by breast cancer patients was associated with reduced risk of recurrence and survival, regardless of whether use was during chemotherapy or not [21]. Because dietary and lifestyle factors may differ between populations, it is unclear as to whether these findings would apply to a mainly white, U.S. population.

There are some limitations in our assessment of physician recommendations. We were unable to discern whether discussions regarding multivitamin and/or supplement use were initiated by physicians or by patients. In addition, the question regarding recommendations from physicians only referred to vitamins and supplements in general, not specific supplements such as antioxidants or those that showed increases in use with chemotherapy. Furthermore, it did not distinguish use of high-dose supplements versus meeting recommended daily requirements. While a recommendation not to take any vitamins and supplements would include antioxidants, a recommendation to use vitamins and supplements may refer to other supplements than antioxidants, such as B vitamins for neuropathy. However, baseline non-users of vitamin $\mathrm{C}$ and $\mathrm{E}$ were more likely to initiate use during treatment after receiving a recommendation to use vitamins and supplements compared to those receiving no recommendation. In this study, regular vitamin $\mathrm{C}$ and $\mathrm{E}$ use, both before diagnosis and during treatment, was relatively low. When stratified by physician recommendation, sample sizes for some groups were small.

We have shown that patterns of use in this study are impacted greatly by physician recommendations. The participants in this clinical trial had "high-risk" Stage II or III breast cancer with large tumors and/or nodal involvement. Patients who enroll in a clinical trial may have more concerns about their prognosis, or may have more reliance upon or confidence in their oncologists, and this could influence the lower use than that reported in the literature. Indeed, our results show that supplement use was most likely to be stopped when patients were advised by their physicians to discontinue use. To our knowledge, this is the first study to report upon patterns of supplement use during chemotherapy in relation to recommendations from treating physicians, and the first survey of these interactions between cancer patients and their doctors. Thus, we are unable to compare our low rates of use of supplements in relation to physician recommendations to other published data on antioxidant use among breast cancer patients. A major strength of our study is the detailed assessment of multivitamin and supplement use at multiple time points, specifically before diagnosis and during breast cancer treatment. The majority of studies reporting on supplement use by cancer patients are derived from data ascertained at diagnosis, or several months following treatment, with little information on patterns during chemotherapy, which could be the most crucial time-point for effects on treatment outcomes.

In this study, a number of participants did not discuss supplement usage with their doctors. This would suggest that better patient-physician communication is needed although there are not clear empirical data upon which to base suggestions regarding habits during chemotherapy. With adequate followup time of patients enrolled in S0221, and detailed information on use of multivitamins and supplements before, during and after chemotherapy, data upon which to base those recommendations will be available for advising patients on habits during cancer therapy.

Acknowledgments We wish to acknowledge and thank Dr. Frank L. Meyskens, Jr., M.D., Associate Chair for the Cancer Control \& Prevention Committees of SWOG, for his administrative support.

Conflict of interest This study was supported by NIH R01 CA116395, and the following PHS Cooperative Agreement Grant numbers awarded by the National Cancer Institute, DHHS: CA32102, CA38926, CA63844, CA63845, CA 20319, CA46282, CA46441, CA35261, CA63848, CA67575, CA14028, CA35281, CA128567, CA45560, CA58882, CA13612; CA46368, CA45808, CA58658, CA76447, CA37981, CA04919; CA95860; CA27057, CA42777, CA22433, CA74647, CA86780, CA68183, CA58861, CA45807, CA35192, CA35178, CA58416, CA35176, CA67663, CA35431, CA12644, CA16385, CA11083, CA45377, CA35128, CA35262, CA52654, CA76429, CA58723, CA46113, CA76132, CA45450, CA35119, CA45461, CA21115, CA21076, CA77597, CA25224, CA77202, CCSRI 15469, and in part by Amgen, Inc. Support was also provided by the Fashion Footwear Charitable Foundation of New York/QVC Presents Shoes on Sale ${ }^{\mathrm{TM}}$ (DFH), and Drs. Ambrosone, Gralow, Hershman and Hortobagyi are recipients of funding from the Breast Cancer Research Foundation. Dr. Budd serves an advisory at Amgen. Dr. Gralow receives funding from Nevartis, Amgen, Genentech, and Roche. Funding agencies had no involvement in the study design, data collection, analysis and interpretation, or in the writing of the report and submission.

Open Access This article is distributed under the terms of the Creative Commons Attribution Noncommercial License which permits any noncommercial use, distribution, and reproduction in any medium, provided the original author(s) and the source are credited.

\section{References}

1. Radimer K, Bindewald B, Hughes J et al (2004) Dietary supplement use by US adults: data from the National Health and 
Nutrition Examination Survey, 1999-2000. Am J Epidemiol 160: 339-349. doi:10.1093/aje/kwh207

2. Rock CL (2007) Multivitamin-multimineral supplements: who uses them? Am J Clin Nutr 85:277-279

3. Rock CL, Newman VA, Neuhouser ML et al (2004) Antioxidant supplement use in cancer survivors and the general population. J Nutr 134:3194-3195

4. Newman V, Rock CL, Faerber S et al (1998) Dietary supplement use by women at risk for breast cancer recurrence. The women's healthy eating and living study group. J Am Diet Assoc 98: 285-292

5. Greenlee H, Gammon MD, Abrahamson PE et al (2009) Prevalence and predictors of antioxidant supplement use during breast cancer treatment: the long Island breast cancer study project. Cancer 115:3271-3282. doi:10.1002/cncr.24378

6. Ambrosone CB, Ahn J, Schoenenberger V (2005) Antioxidant supplements, genetics and chemotherapy outcomes. Curr Cancer Ther Rev I:251-258

7. Lawenda BD, Kelly KM, Ladas EJ et al (2008) Should supplemental antioxidant administration be avoided during chemotherapy and radiation therapy? J Natl Cancer Inst 100:773-783. doi: 10.1093/jnci/djn148

8. Norman HA, Butrum RR, Feldman E et al (2003) The role of dietary supplements during cancer therapy. J Nutr 133:3794-3799

9. American Institute for Cancer Research (2000) Nutrition of the cancer patient. Washington, DC

10. Cassileth BR, Vickers AJ (2005) High prevalence of complementary and alternative medicine use among cancer patients: implications for research and clinical care. J Clin Oncol 23: 2590-2592. doi:10.1200/JCO.2005.11.922

11. Greenlee H, Kwan ML, Kushi LH et al (2012) Antioxidant supplement use after breast cancer diagnosis and mortality in the life after cancer epidemiology (LACE) cohort. Cancer 118: 2048-2058. doi:10.1002/cncr.26526

12. Mehta DH, Gardiner PM, Phillips RS et al (2008) Herbal and dietary supplement disclosure to health care providers by individuals with chronic conditions. J Altern Complement Med 14: 1263-1269. doi:10.1089/acm.2008.0290

13. Ashikaga T, Bosompra K, O'Brien P et al (2002) Use of complementary and alternative medicine by breast cancer patients: prevalence, patterns and communication with physicians. Supportive care in cancer: official journal of the multinational association of supportive care in cancer 10:542-548. doi:10.1007/ s00520-002-0356-1

14. Rausch SM, Winegardner F, Kruk KM et al (2011) Complementary and alternative medicine: use and disclosure in radiation oncology community practice. Supportive care in cancer: official journal of the multinational association of supportive care in cancer 19:521-529. doi:10.1007/s00520-010-0846-5

15. Ferrucci LM, McCorkle R, Smith T et al (2009) Factors related to the use of dietary supplements by cancer survivors. J Altern Complement Med 15:673-680. doi:10.1089/acm.2008.0387

16. Kwan D, Hirschkorn K, Boon H (2006) U.S. and Canadian pharmacists' attitudes, knowledge, and professional practice behaviors toward dietary supplements: a systematic review. BMC Complement Altern Med 6:31. doi:10.1186/1472-6882-6-31

17. Roth M, Lin J, Kim M et al (2009) Pediatric oncologists' views toward the use of complementary and alternative medicine in children with cancer. Journal of Pediatric Hematology/Oncology: official journal of the American Society of Pediatric Hematology/ Oncology 31:177-182. doi:10.1097/MPH.0b013e3181984f5a

18. Satia-Abouta J, Patterson RE, King IB et al (2003) Reliability and validity of self-report of vitamin and mineral supplement use in the vitamins and lifestyle study. Am J Epidemiol 157:944-954. doi:10.1093/aje/kwg039

19. Greenlee H, Hershman DL, Jacobson JS (2009) Use of antioxidant supplements during breast cancer treatment: a comprehensive review. Breast Cancer Res Treat 115:437-452. doi:10.1007/ s10549-008-0193-0

20. $\mathrm{Ng} \mathrm{K}$, Meyerhardt JA, Chan JA et al (2010) Multivitamin use is not associated with cancer recurrence or survival in patients with stage III colon cancer: findings from CALGB 89803. Journal of Clinical Oncology: official journal of the American Society of Clinical Oncology 28:4354-4363. doi:10.1200/JCO.2010.28.0362

21. Nechuta S, Lu W, Chen Z et al (2011) Vitamin supplement use during breast cancer treatment and survival: a prospective cohort study. Cancer Epidemiol Biomarkers Prev 20:262-271. doi:10. 1158/1055-9965.EPI-10-1072 